\title{
The Newtonian Limit of the Spherically Symmetric Vlasov-Einstein System
}

\author{
G. Rein ${ }^{1}$ and A.D. Rendall ${ }^{2}$ \\ 1 Mathematisches Institut der Universität München, Theresienstr. 39, W-8000 München 2, \\ Germany \\ 2 Max-Planck-Institut für Astrophysik, Karl-Schwarzschild-Str. 1, W-8046 Garching, Germany
}

Received March 25, 1992

Abstract. We prove that spherically symmetric solutions of the Vlasov-Einstein
system with a fixed initial value converge to the corresponding solution of the
Vlasov-Poisson system if the speed of light $c$ is taken as a parameter and tends to
infinity. The convergence is uniform on compact time intervals with convergence
rate $1 / c^{2}$. Thus the classical Vlasov-Poisson system appears as the Newtonian limit
of the general relativistic Vlasov-Einstein system in a spherically symmetric setting.

\section{Introduction}

Consider an ensemble of particles (stars in a galaxy, galaxies in a galaxy cluster, etc.) which are all of the same mass and interact only by the gravitational field which they generate collectively. The ensemble is described by a time dependent density function $f$ on phase space, and this function satisfies a continuity equation, the Vlasov or Liouville equation, which is coupled to the field equations with source terms generated by $f$. In the classical, Newtonian setting we obtain the Vlasov-Poisson system (VP)

$$
\begin{gathered}
\partial_{t} f+v \cdot \partial_{x} f-K(t, x) \cdot \partial_{v} f=0, \\
K(t, x):=\frac{x}{r} \frac{1}{r^{2}} \int_{|y| \leqq r} \int_{\mathbb{R}^{3}} f(t, y, v) d v d y,
\end{gathered}
$$

where $t \geqq 0$ denotes time, $x \in \mathbb{R}^{3}$ position, and $v \in \mathbb{R}^{3}$ momentum, $r:=|x|$, and we have assumed that the system is spherically symmetric, i.e. $f(t, A x, A v)=f(t, x, v)$, $K(t, A x)=A K(t, x)$ for any orthogonal matrix $A$ and $t \geqq 0, x, v \in \mathbb{R}^{3}$.

If we wish to describe the above situation in the setting of general relativity, we obtain the Vlasov-Einstein system (VE $\gamma$ ) in the following form:

$$
\begin{gathered}
\partial_{t} f+e^{\mu-\lambda} \frac{v}{\sqrt{1+\gamma v^{2}}} \cdot \partial_{x} f-\left(\dot{\lambda} \frac{x \cdot v}{r}+e^{\mu-\lambda} \frac{1}{\gamma} \mu^{\prime} \sqrt{1+\gamma v^{2}}\right) \frac{x}{r} \cdot \partial_{v} f=0, \\
e^{-2 \lambda}\left(2 r \lambda^{\prime}-1\right)+1=8 \pi \gamma r^{2} \rho, \\
e^{-2 \lambda}\left(2 r \mu^{\prime}+1\right)-1=8 \pi \gamma^{2} r^{2} p,
\end{gathered}
$$


where

$$
\begin{aligned}
& \rho(t, x):=\int_{\mathbb{R}^{3}} f(t, x, v) \sqrt{1+\gamma v^{2}} d v, \\
& p(t, x):=\int_{\mathbb{R}^{3}} f(t, x, v)\left(\frac{x \cdot v}{r}\right)^{2} \frac{d v}{\sqrt{1+\gamma v^{2}}} .
\end{aligned}
$$

Here the meaning and range of the variables $t, x, v$ is as above, $\gamma:=1 / c^{2}$, where $c$ denotes the speed of light, and ${ }^{-}$and ' denote the partial derivative with respect to $t$ and $r=|x|$ respectively. If we let $x=r(\sin \theta \cos \phi, \sin \theta \sin \phi, \cos \theta)$ then the space-time metric is given by

$$
d s^{2}=-e^{2 \mu} d t^{2}+\gamma\left(e^{2 \lambda} d r^{2}+r^{2}\left(d \theta^{2}+\sin ^{2} \theta d \phi^{2}\right)\right) .
$$

We refer to [9] as to how (VEy) is obtained from the full Vlasov-Einstein system without symmetry, i.e. on the particular choice of coordinates and the meaning of spherical symmetry in the setting of general relativity. In the above coordinates, spherical symmetry can be defined as for the Vlasov-Poisson system and results in the fact that $\lambda, \mu, \rho, p$ can be seen as functions of $t \geqq 0$ and $r \geqq 0$ or $t \geqq 0$ and $x \in \mathbb{R}^{3}$.

Both systems are supplemented by the initial condition $f(0)=\stackrel{\circ}{f}$ with a nonnegative, spherically symmetric function $\stackrel{\circ}{f}$, and for $(\mathrm{VE} \gamma)$ we require asymptotic flatness of space-time and a regular center at $r=0$ which leads to the boundary conditions

$$
\lim _{r \rightarrow \infty} \lambda(t, r)=\lim _{r \rightarrow \infty} \mu(t, r)=\lambda(t, 0)=0, \quad t \geqq 0 .
$$

The purpose of the present note is to prove that for a fixed initial distribution $f$ the solutions of (VE $\gamma)$ tend to the corresponding solution of (VP) for $\gamma \rightarrow 0$. In order for this statement to make sense we also have to show that all solutions in question exist on an $\gamma$-independent time interval. The convergence is uniform on such compact time intervals where all solutions exist, and the rate of convergence is $\gamma$. As far as we know, this is the first result (with the exception of certain static and explicit solutions, cf. [4]) where the Newtonian limit of the general relativistic equations is not only shown formally in the sense that the equations pass to their Newtonian equivalent with $c \rightarrow \infty$ but where the convergence of the corresponding solutions is established as well. In passing we note that by a solution we always mean a sufficiently regular function which satisfies the equations classically.

The paper proceeds as follows: In the next section we formulate and briefly discuss our main results. In the third section we establish the existence of a time interval where all solutions in question exist and satisfy certain uniform bounds which are then used in the last section to pass to the limit $\gamma \rightarrow 0$.

We conclude this introduction with some remarks on the literature. The existence of global, classical solutions to the initial value problem for (VP) without smallness or symmetry assumptions on the data is shown in [7], cf. also $[2,5,6,11]$. For (VE $\gamma)$ we refer to [9] where we established a local existence and uniqueness result together with a criterion for global existence and proved that solutions are indeed global for sufficiently small initial data. To our knowledge this is the first global existence result for Einstein's field equations coupled to any kind of description of matter. For further references we refer to [9]. The present 
investigation is also related to [10], where the classical limit of the relativistic Vlasov-Maxwell system is investigated, cf. also $[1,3]$. As to the existence theory of the latter system we refer to [8] and the references therein.

\section{Main Results}

Let us first fix the set of initial data which we consider:

$$
\begin{aligned}
\mathscr{D}:= & \left\{g \in C^{1}\left(\mathbb{R}^{3} \times \mathbb{R}^{3}\right) \mid g \geqq 0 \text {, supp } g \text { compact, } g\right. \text { spherically symmetric, } \\
& \left.\frac{1}{r} \int_{|x|<r} \int_{\mathbb{R}^{3}} g(x, v) \sqrt{1+v^{2}} d v d x<\frac{1}{2}, r>0\right\} .
\end{aligned}
$$

Using the local existence result and the continuation criterion established in [9] it will be shown that the solutions of $(\mathrm{VE} \gamma)$ exist on an interval $[0, T[$ which is independent of $\gamma$; more precisely:

Theorem 1. Let $\stackrel{\circ}{f} \in \mathscr{D}$. Then there exists $T>0$ and $u:\left[0, T\left[\rightarrow \mathbb{R}^{+}\right.\right.$continuous such that for $\gamma \in] 0,1]$ the system (VE $\gamma$ ) has a classical solution $\left(f_{\gamma}, \lambda_{\gamma}, \mu_{\gamma}\right)$ on the interval $\left[0, T\left[\right.\right.$ with $f_{\gamma}(0)=f$ and

$$
f_{\gamma}(t, x, v)=0, \quad|v|>u(t), \quad x \in \mathbb{R}^{3}, \quad t \in[0, T[, \quad \gamma \in] 0,1] .
$$

At least on the interval [0,T[ it now makes sense to investigate the limit $\gamma \rightarrow 0$. The following theorem is the main result of the present paper.

Theorem 2. Let $\stackrel{\circ}{f} \in \mathscr{D}$ and let $0<T \leqq \infty$ be such that $\left(f_{\gamma}, \lambda_{\gamma}, \mu_{\gamma}\right)$ exists on $[0, T[$ for all $\gamma \in] 0,1]$ and

$$
f_{\gamma}(t, x, v)=0, \quad|v|>u(t), \quad x \in \mathbb{R}^{3}, \quad t \in[0, T[, \quad \gamma \in] 0,1]
$$

for a continuous function $u:\left[0, T\left[\rightarrow \mathbb{R}^{+}\right.\right.$. Let $f \in C^{1}\left(\left[0, \infty\left[\times \mathbb{R}^{6}\right)\right.\right.$ denote the solution of (VP) with $f(0)=f$. Then for any $\left.T^{\prime} \in\right] 0, T[$ there exists a constant $C>0$ such that for all $\gamma \in] 0,1]$ the following estimate holds:

$$
\begin{gathered}
\left\|f_{\gamma}(t)-f(t)\right\|_{\infty}+\left\|\lambda_{\gamma}(t)\right\|_{\infty}+\left\|\mu_{\gamma}(t)\right\|_{\infty}+\left\|\dot{\lambda}_{\gamma}(t)\right\|_{\infty} \\
+\left\|\frac{1}{\gamma} \partial_{x} \mu_{\gamma}(t)-K(t)\right\|_{\infty} \leqq C \gamma, \quad t \in\left[0, T^{\prime}\right] .
\end{gathered}
$$

Theorem 1 shows that the assumptions in Theorem 2 are indeed satisfied on some interval $[0, T[$. The reason for separating the two results is the following: In the general situation of Theorem 1 the interval may be quite short. On the other hand it is possible that for more restricted initial data the assumptions in Theorem 2 are even satisfied on $[0, \infty$ [, cf. the global existence result for small data in [9].

\section{Proof of Theorem 1}

First note that for $\gamma \in] 0,1]$ the estimate

$$
\gamma \frac{1}{r} \int_{|x|<r} \int_{\mathbb{R}^{3}} f(x, v) \sqrt{1+\gamma v^{2}} d v d x<\frac{1}{2}, \quad r>0
$$


holds so that by the local existence result in [9] we obtain a solution $\left(f_{\gamma}, \lambda_{\gamma}, \mu_{\gamma}\right)$ on a maximal existence interval $\left[0, T_{\gamma}\left[, T_{\gamma}>0\right.\right.$, with $f_{\gamma}(0)=f$; actually, in [9] we restricted ourselves to the case $\gamma=1$. However, if $(f, \lambda, \mu)$ is a solution of (VE1), then $\left(\gamma^{3 / 2} f\left(\cdot, \gamma^{1 / 2} \cdot, \gamma^{1 / 2} \cdot\right), \lambda\left(\cdot, \gamma^{1 / 2} \cdot\right), \mu\left(\cdot, \gamma^{1 / 2} \cdot\right)\right)$ solves (VE $\left.\gamma\right)$. Next we recall that integrating the field equations subject to the boundary conditions yields

$$
e^{-2 \lambda_{y}(t, r)}=1-2 \gamma \frac{m_{y}(t, r)}{r}
$$

and

$$
\mu_{\gamma}(t, r)=-\int_{r}^{\infty} e^{2 \lambda_{\gamma}(t, s)}\left(\gamma \frac{m_{\gamma}(t, s)}{s^{2}}+4 \pi \gamma^{2} s p_{\gamma}(t, s)\right) d s
$$

where

$$
m_{\gamma}(t, r):=4 \pi \int_{0}^{r} s^{2} \rho_{\gamma}(t, s) d s=\int_{|y| \leqq r} \rho_{\gamma}(t, y) d y ;
$$

cf. [9]. If we let $X_{\gamma}(\cdot, t, x, v), V_{\gamma}(\cdot, t, x, v)$ denote the solution of the characteristic system

$$
\begin{aligned}
\dot{x} & =e^{\mu_{\gamma}-\lambda_{\gamma}} \frac{v}{\sqrt{1+\gamma v^{2}}}, \\
\dot{v} & =-\left(\dot{\lambda}_{\gamma} \frac{x \cdot v}{r}+e^{\mu_{\gamma}-\lambda_{\gamma}} \frac{1}{\gamma} \mu_{\gamma}^{\prime} \sqrt{1+\gamma v^{2}}\right) \frac{x}{r},
\end{aligned}
$$

with $X_{\gamma}(t, t, x, v)=x, V_{\gamma}(t, t, x, v)=v$, then

$$
f_{\gamma}(t, x, v)=\stackrel{\circ}{f}\left(X_{\gamma}(0, t, x, v), V_{\gamma}(0, t, x, v)\right) .
$$

Among other things this implies that $\left\|f_{\gamma}(t)\right\|_{\infty}=\|\stackrel{\circ}{f}\|_{\infty}, t \geqq 0$. We have the estimates

$$
\begin{aligned}
&\left\|\rho_{\gamma}(t)\right\|_{\infty} \leqq C u_{\gamma}^{3}(t) \sqrt{1+u_{\gamma}^{2}(t)}, \\
&\left\|p_{\gamma}(t)\right\|_{\infty} \leqq C \min \left\{\frac{1}{\sqrt{\gamma}} u_{\gamma}^{4}(t), u_{\gamma}^{5}(t)\right\},
\end{aligned}
$$

where $C$ denotes a constant which does not depend on $\gamma$ or $t$ and may change from line to line and

$$
u_{\gamma}(t):=\sup \left\{|v| \mid(x, v) \in \operatorname{supp} f_{\gamma}(t)\right\}
$$

The estimate for $\rho_{\gamma}$ implies that

$$
\begin{aligned}
& \frac{m_{\gamma}(t, r)}{r} \leqq C u_{\gamma}(t) \sqrt{1+u_{\gamma}^{2}(t)}, \\
& \frac{m_{\gamma}(t, r)}{r^{2}} \leqq C u_{\gamma}^{2}(t) \sqrt{1+u_{\gamma}^{2}(t)} .
\end{aligned}
$$


Using this information we can estimate $e^{\mu_{\gamma}-\lambda_{\gamma}} \mu_{\gamma}^{\prime}$ and $\dot{\lambda}_{\gamma}$. From

$$
e^{\mu_{\gamma}-\lambda_{y}} \mu_{\gamma}^{\prime}(t, r)=e^{\mu_{\gamma}+\lambda_{\gamma}}\left(\gamma \frac{m_{\gamma}(t, r)}{r^{2}}+4 \pi \gamma^{2} r p_{\gamma}(t, r)\right)
$$

we conclude that

$$
\left|\frac{1}{\gamma} e^{\mu_{\gamma}-\lambda_{y}} \mu_{\gamma}^{\prime}(t, r)\right| \leqq C(1+t)\left(1+u_{\gamma}(t)\right)^{4} ;
$$

note that along a characteristic we have $|\dot{x}|=|v| / \sqrt{1+\gamma v^{2}} \leqq 1 / \sqrt{\gamma}$ so that $r=$ $|x| \leqq r_{0}+t / \sqrt{\gamma}$ for $(x, v) \in \operatorname{supp} f_{\gamma}(t)$. Furthermore, the fact that $\lambda_{\gamma}^{\prime}+\mu_{\gamma}^{\prime} \leqq 0$ and the boundary conditions at $r=\infty$ imply that $\lambda_{\gamma}+\mu_{\gamma} \leqq 0$. As is shown in [9] not only the two Einstein equations given above hold for our solution but all other Einstein equations hold as well. In particular.

$$
\dot{\lambda}_{\gamma}(t, r)=-4 \pi \gamma r e^{\lambda_{\gamma}+\mu_{\gamma}} j_{\gamma}(t, r),
$$

where

$$
j_{\gamma}(t, r):=\int f_{\gamma}(t, x, v) \frac{x \cdot v}{r} d v
$$

so that

$$
\left\|j_{\gamma}(t)\right\|_{\infty} \leqq C\left(1+u_{\gamma}(t)\right)^{4}
$$

which implies

$$
\left\|\dot{\lambda}_{\gamma}(t)\right\|_{\infty} \leqq C\left\{\begin{array}{l}
(1+t)\left(1+u_{\gamma}(t)\right)^{4} \\
\gamma(1+t)\left(1+u_{\gamma}(t)\right)^{5}
\end{array} .\right.
$$

The second line in the estimate follows from the observation that $|\dot{x}| \leqq u_{\gamma}(t)$ for characteristics in the support of $f_{\gamma}$. If we insert the above estimates in the characteristic system and integrate we obtain the estimate

$$
u_{\gamma}(t) \leqq u_{0}+C \int_{0}^{t}(1+s)\left(1+u_{\gamma}(s)\right)^{5} d s, \quad t \in\left[0, T_{\gamma}[,\right.
$$

where $u_{0}$ is a bound for $|v|$ on supp $\stackrel{\circ}{f}$. Let $u:\left[0, T\left[\rightarrow \mathbb{R}^{+}\right.\right.$be the maximal solution of the initial value problem

$$
\dot{u}(t)=C(1+t)(1+u(t))^{5}, \quad u(0)=u_{0} .
$$

Now $u_{\gamma}(t) \leqq u(t)$ on $\left[0, T\left[\cap\left[0, T_{\gamma}[\right.\right.\right.$, and the continuation criterion established in [9] implies that $T_{\gamma} \geqq T$ and $u_{\gamma}(t) \leqq u(t)$ on [0,T [, and the proof of Theorem 1 is complete.

\section{Proof of Theorem 2}

Let $0<T^{\prime}<T$. The assumption together with the estimates obtained above imply that there exists a constant $C>0$ such that

$$
\left.\left.u_{\gamma}(t), e^{2 \lambda_{\gamma}(t, r)}, \quad \frac{m_{\gamma}(t, r)}{r}, \frac{m_{\gamma}(t, r)}{r^{2}} \leqq C, \quad t \in\left[0, T^{\prime}\right], \quad r>0, \quad \gamma \in\right] 0,1\right] ;
$$


note that

$$
e^{-2 \lambda_{y}(0, r)}=1-2 \gamma \frac{m_{\gamma}(0, r)}{r} \geqq 1-2 \frac{m_{1}(0, r)}{r}>0, \quad r>0,
$$

and $m_{1}(0, r)=m_{1}\left(0, r_{0}\right)$ for $r>r_{0}$ which implies that

$$
\left.\left.e^{2 \lambda_{y}(0, r)}<C, \quad \gamma \in\right] 0,1\right], \quad r>0,
$$

where $r_{0}$ is a bound for $|x|$ on supp $f$. Together with Eq. (4) this yields the estimate for $e^{2 \lambda_{y}(t, r)}$. Equation (1) implies that

$$
\left|e^{-2 \lambda_{y}(t, r)}-1\right| \leqq C \gamma
$$

and thus

$$
\left\|\lambda_{\gamma}(t)\right\|_{\infty} \leqq \mathrm{C} \gamma, \quad t \in\left[0, T^{\prime}\right]
$$

Equation (4) yields

$$
\left\|\dot{\lambda}_{\gamma}(t)\right\|_{\infty} \leqq C \gamma, \quad t \in\left[0, T^{\prime}\right] .
$$

Equation (2) together with the uniform estimate for the support of $f_{\gamma}(t)$ on $\left[0, T^{\prime}\right]$ implies that

$$
\left\|\mu_{\gamma}(t)\right\|_{\infty} \leqq C \gamma, \quad t \in\left[0, T^{\prime}\right]
$$

and thus

$$
\left|e^{\mu_{y}(t, r)-\lambda_{y}(t, r)}-1\right| \leqq C \gamma, \quad t \in\left[0, T^{\prime}\right], \quad r \geqq 0
$$

Finally,

$$
\begin{aligned}
\left|\frac{1}{\gamma} \partial_{x} \mu_{\gamma}(t, x)-K(t, x)\right| \leqq & \left|e^{2 \lambda_{\gamma}(t, r)} 4 \pi \gamma r p_{\gamma}(t, r)\right|+\left|e^{2 \lambda_{\gamma}(t, r)}-1\right| \frac{m_{\gamma}(t, r)}{r^{2}} \\
& +\left|\frac{m_{\gamma}(t, r)}{r^{2}}-\frac{m(t, r) \mid}{r^{2}}\right| \\
\leqq & C \gamma+\frac{1}{r^{2}}\left|m_{\gamma}(t, r)-m(t, r)\right| \\
\leqq & C \gamma+C\left\|f_{\gamma}(t)-f(t)\right\|_{\infty} .
\end{aligned}
$$

Now

$$
\begin{aligned}
\left|f_{\gamma}-f\right|(t, x, v) & =\left|\stackrel{\circ}{f}\left(X_{\gamma}(0, t, x, v), V_{\gamma}(0, t, x, v)\right)-\stackrel{\circ}{f}(X(0, t, x, v), V(0, t, x, v))\right| \\
& \leqq C \alpha_{\gamma}(t)
\end{aligned}
$$

where

$$
\begin{gathered}
\alpha_{\gamma}(t):=\sup \left\{\left|X_{\gamma}-X\right|(0, t, x, v)+\left|V_{\gamma}-V\right|(0, t, x, v) \mid\right. \\
\left.(x, v) \in \operatorname{supp} f_{\gamma}(t) \cup \operatorname{supp} f(t)\right\} .
\end{gathered}
$$

From the characteristic systems for (VE $\gamma$ ) and (VP) respectively we can now derive a differential inequality for $\left|X_{\gamma}-X\right|(0, t, x, v)+\left|V_{\gamma}-V\right|(0, t, x, v)$. Inserting the 
estimates' (5), (6), (7), (8), and (9) into this inequality, observing that $\partial_{x} K$ is bounded on $\left[0, T^{\prime}\right] \times \mathbb{R}^{3}$, and that

$$
\left|\frac{V_{\gamma}}{\sqrt{1+\gamma V_{\gamma}^{2}}}-V\right| \leqq\left|V_{\gamma}-V\right|+\left|\sqrt{1+\gamma V_{\gamma}^{2}}-1\right| \leqq\left|V_{\gamma}-V\right|+C \gamma
$$

we obtain, after integrating with respect to time, the estimate

$$
\alpha_{\gamma}(t) \leqq C \gamma+C \int_{0}^{t} \alpha_{\gamma}(s) d s, \quad t \in\left[0, T^{\prime}\right]
$$

where $C$ depends on $T^{\prime}$ but not on $\gamma$. Thus by Gronwall's inequality

$$
\left.\left.\alpha_{\gamma}(t) \leqq C \gamma, \quad t \in\left[0, T^{\prime}\right], \quad \gamma \in\right] 0,1\right],
$$

and together with the above estimates this completes the proof of Theorem 2 .

\section{References}

1. Asano, K., Ukai, S.: On the Vlasov-Poisson limit of the Vlasov-Maxwell equations. Stud. Math. Appl. 18, 369-383 (1986)

2. Batt, J., Rein, G.: Global classical solutions of the periodic Vlasov-Poisson system in three dimensions. C. R. Acad. Sc. Paris. 313, 411-416 (1991)

3. Degond, P.: Local existence of solutions of the Vlasov-Maxwell equations and convergence to the Vlasov-Poisson equations for infinite light velocity. Math. Meth. Appl. Sci. 8, 533-558 (1986)

4. Ehlers, J.: The Newtonian limit of general relativity. In: Ferrarese, G. (ed.) Classical mechanics and relativity: Relationship and consistency. Naples: Bibliopolis 1991

5. Horst, E.: On the asymptotic growth of the solutions of the Vlasov-Poisson system. Preprint 1991

6. Lions, P.-L., Perthame, B.: Propagation of moments and regularity for the 3-dimensional Vlasov-Poisson system. Invent. Math. 105, 415-430 (1991)

7. Pfaffelmoser, K.: Global classical solutions of the Vlasov-Poisson system in three dimensions for general initial data. J. Diff. Eqns. 95, 281-303 (1992)

8. Rein, G.: Generic global solutions of the relativistic Vlasov-Maxwell system of plasma physics. Commun. Math. Phys. 135, 41-78 (1990)

9. Rein, G., Rendall, A.: Global existence of solutions of the spherically symmetric VlasovEinstein system with small initial data. Commun. Math. Phys. 150, 561-583 (1992)

10. Schaeffer, J.: The classical limit of the relativistic Vlasov-Maxwell system. Commun. Math. Phys. 104, 403-421 (1986)

11. Schaeffer, J.: Global existence of smooth solutions to the Vlasov-Poisson system in three dimensions. Commun. Part. Diff. Eqns. 16, 1313-1335 (1991) 
